\title{
Evaluation and Ranking of Organizational Resilience Factors by Using a Two-Step Fuzzy AHP and Fuzzy TOPSIS
}

\author{
Danijela Tadić, Aleksandar Aleksić, Miladin Stefanović, and Slavko Arsovski \\ Faculty of Engineering, University of Kragujevac, Sestre Janjic 6, 34000 Kragujevac, Serbia \\ Correspondence should be addressed to Miladin Stefanović; miladin@kg.ac.rs
}

Received 9 September 2013; Accepted 25 December 2013; Published 19 February 2014

Academic Editor: Wudhichai Assawinchaichote

Copyright ( 2014 Danijela Tadić et al. This is an open access article distributed under the Creative Commons Attribution License, which permits unrestricted use, distribution, and reproduction in any medium, provided the original work is properly cited.

\begin{abstract}
We presented a novel fuzzy multicriteria decision making approach to evaluate and rank organizational resilience factors with respect to user preference orders. Due to vagueness of the decision data, the precise numerical data are inadequate for real-life business situations. Human judgements can be expressed by linguistic expressions which are modeled by fuzzy sets. The complexity of the considered problem calls for analytic methods rather than intuitive decisions. Two fuzzy multi-criteria methods are proposed for solving the treated problem: Fuzzy Analytic Hierarchical Process (FAHP) is applied to determine the relative importance of business processes and the relative importance of organizational resilience factors under each business process, and an extension of the fuzzy Technique for Order Preference by Similarity to Ideal Solution (FTOPSIS) is applied to rank the organizational resilience factors. With respect to complexity and the type of considered management problem, we introduce a modified fuzzy decision matrix. The proposed algorithm has efficiently been applied in the assessment of organizational resilience factors to small and medium enterprises of the process industry.
\end{abstract}

\section{Introduction}

In the last few decades, theory and good practice of management have shown that the concept of organizational resilience has been employed in order to establish mechanisms for overcoming crisis and emerging disorder. Organizational resilience includes the ability of an organization to withstand systematic discontinuities, and the capability to adapt to new environments has emerged from different risk sources [1]. The different issues, such as the size of an organization, economy branch, or supply chain positions, have not allowed the setting of a scientific consensus nor the constituent elements of organizational resilience. Market demands often introduce complexity and variable business conditions where the sources of risk need to be managed in the long term, in order to ensure sustainability and enhance overall quality [2]. A high competitive edge can be achieved by applying an adequate business strategy which may be based on the ranking of organizational resilience factors [3]. The organizational resilience potential has to be continuously enhanced and its value may be analysed through the appropriate resilience factors.

In this paper, the problem of evaluation and ranking of organizational resilience factors set in small and medium enterprises (SMEs) of the process industry is considered. SMEs that belong to the process industry may produce different products, for example, mining and mineral, food and beverage, pulp and paper, chemical products, basic metal, or other process industry products. SMEs are recognized as an important sector in the industry of developing countries or as the policy in developed countries aiming to stimulate entrepreneurship in Europe where they form the backbone of the EU economy accounting for 99.8 per cent of nonfinancial enterprises in 2012, which equates to 20.7 million businesses [4]. Clients' expectations all over the world have significantly increased during the last decade, so SMEs have to be innovative and adapt to new challenges [5] by combining old and new business models and organizational resilience enhancement. Having in mind the interconnected nature of business and production processes in this type of industry, 
it is clear that achieving resilience of such organizations is very important issue. The motivation for this work came from the fact that there is no accepted model for ranking organizational resilience factors in one organization, since there are no universal organizational resilience assessment models [6].

Uncertainties in the relative importance of business processes, the relative importance of organizational resilience factors with respect to each business process, and organizational resilience values are judged by decision makers. Decision makers express their judgements far better by using linguistic expressions than by representing them in terms of precise numbers. In this paper, modeling of linguistic expressions is based on the fuzzy set theory $[7,8]$. Fuzzy set theory supports the subjective natural language descriptors of organizational resilience and it provides a methodology for allowing subjective natural language descriptors to enter into the modeling process. The fuzzy set theory resembles human reasoning in its use of approximate information and uncertainty to generate decisions.

The treated issue can be stated as a multicriteria optimization problem task. One of the most outstanding multicriteria methods is the Analytic Hierarchical Process (AHP) [9] and the Technique for Order Preference by Similarity to Ideal Solution (TOPSIS) [10]. In many papers, which can be found in the literature, the different multicriteria problems are solved by the proposed two-stage method. At the first stage, either the AHP or fuzzy AHP (FAHP) method is used to determine the weight of the treated criteria [11-13]. At the second stage, TOPSIS or fuzzy TOPSIS (FTOPSIS) is used in order to determine the best alternative with respect to all the treated criteria, simultaneously, as well as their relative importance. In the literature, there are numerous applications of two-stage methods within the several aspects of management.

The main objective of this work is to determine the rank of organizational resilience factors on the level of enterprise (SME) by using the proposed FTOPSIS. This should enable a wide base for benchmarking the similar organizations and the enhancement of their overall resilience potential.

The paper is organized in the following way. Section 2 gives a brief literature review in the analysed scientific field. Section 3 reviews the concepts of the proposed analytic method framework. The handling of uncertainties in the relative importance of business processes, the relative importance of organizational resilience factors with respect to each business process, and organizational resilience factor values are presented in Section 4. In Section 5, we present an algorithm to extend TOPSIS to deal with fuzzy data. Section 6 presents an empirical application of the proposed method for the selection of organizational resilience factors in the process industry. Finally, a discussion of research and conclusions are presented in Section 7.

\section{Related Work}

In order to keep the assessment consistent during a defined period of time, the ranking should be done on the level of organizational units with assets that can be influenced by managers [3]. There are a lot of variables that may influence resilience of the identified critical processes [14] and the overall resilience of an organization.

A brief literary review of papers dealing with the evaluation and ranking of different items by the fuzzy AHP and fuzzy TOPSIS approaches is given further in the paper. In [15] the determining of criteria weights and alternative values are stated as group decision making problems. The fuzzy rating of these values is described by five predefined linguistic expressions which are modeled by triangular fuzzy numbers which are defined in interval [0-1]. By using the linear normalization method [16], the normalized decision matrix is obtained. FPISs and FNISs are calculated by applying the six different approaches presented in the literature. The rank of alternatives is obtained by using a comparison of each alternative with the veto threshold defined for each criterion. In [11] the performance rating of each alternative with respect to each criterion and the fuzzy rating of criteria weights are described by five linguistic expressions which are modeled by triangular fuzzy numbers. These triangular fuzzy numbers are defined on a real line in interval [0-1]. The normalized values of alternatives with respect to the criteria type are obtained by applying the normalized procedure [17]. The FPISs and FNISs of the fuzzy weighted normalized matrix are calculated by using max. and min. operators. The separation measures are calculated by using an expression for determining the distance between two triangular fuzzy numbers. The closeness coefficient which is associated with each alternative is obtained as crisp numerical data.

In [18], the criteria weights are obtained by assessment of decision makers by consensus. The criteria weights are described by five linguistic expressions which are modeled by triangular fuzzy numbers which belong to interval [0-1] of a real set. The performance rating of alternatives with respect to each criterion is stated as a group decision making problem. Decision makers used seven linguistic expressions for the fuzzy rating of alternatives. These linguistic expressions are modeled by triangular fuzzy numbers which belong to interval [1-10]. The fuzzy performance score of each alternative is calculated by using the average value method. The normalized fuzzy alternative values are obtained by applying the linear normalization procedure [16]. FPISs and FNISs are calculated according to the procedure which is proposed in this paper. In [19], a fuzzy TOPSIS approach based on a modified preference ratio and an efficient fuzzy distance measurement is proposed. The fuzzy rating of criteria weights and preference alternatives with respect to each criterion is performed by experts. The criteria weights can be described by nine linguistic expressions which are modeled by trapezoidal fuzzy numbers in interval [0-1]. The preference alternatives with respect to each criterion are described by linguistic expressions which are modeled by trapezoidal fuzzy numbers in interval [1-10]. The normalized values of alternatives are given by using the linear normalization procedure [16]. The FPISs and FNISs are calculated by using max. and min. operators. The fuzzy distances of each alternative from the FPISs and FNISs are known as the positive fuzzy distance (PFD) and the negative fuzzy distance 
(NFD). The calculation of PFD and NFD is based on using the extended fuzzy distance measurement for trapezoidal fuzzy numbers which has been proven in this paper. A novel fuzzy distance measurement method was proposed for generalized fuzzy numbers by C. Chakraborty and D. Chakraborty [20]. The ranking of alternatives with respect to all criteria and their weights is performed by applying the initial estimation method [21]. Gumus [22] presented the criteria according to which hazardous waste transportation firms are determined by a modified Delphi method. The fuzzy pairwise comparison matrix of the relative importance of criteria is stated. The vector of criteria weights is calculated by using FAHP [23]. The alternative values are described by precise numbers. The normalization of elements of the decision matrix is performed by using the normalization procedure of conventional TOPSIS. PISs and NISs are determined with respect to the weighted normalized decision matrix. The separation measures are calculated by using $n$-dimensional Euclidean distance. The ranking of alternatives using closeness coefficients is calculated as in conventional TOPSIS. In Tadić et al. [12], three decision makers use five linguistic expressions to describe the relative importance of each pair of treated criteria which are modeled by triangular fuzzy numbers. These triangular fuzzy numbers are defined on the common measurement scale (by analogy to AHP). The fuzzy rating of each decision maker is modeled by triangular fuzzy numbers. The aggregated values of the relative importance of each pair of criteria are given by the proposed method. The vector of criteria weights is calculated by using the extent analysis method [23]. The criteria values can be crisp and uncertain. The normalization of crisp criteria is performed according to the normalization procedure which is defined in the conventional TOPSIS method. Uncertain criteria values are described by triangular fuzzy numbers in the interval [0-1]. FPISs and FNISs are determined by using a method for the comparison of fuzzy numbers [24]. A closeness coefficient according to which a rank of alternatives is determined is calculated using a procedure defined in the conventional TOPSIS method.

In the literature there are many papers which can be found in which fuzzy TOPSIS is proposed. The main differences between the different proposed fuzzy TOPSIS approaches can be summarized in the choice of the number of linguistic expressions for the describing of uncertain variables, in the choice of membership functions and domains of fuzzy numbers for modeling of linguistic expressions, in the choice of the normalization method of the fuzzy decision matrix, in the determination of FPISs and FNISs, and in the distance calculation between fuzzy numbers and the calculation of closeness coefficients values. Most of the proposed fuzzy TOPSIS used a defuzzification method during the early or middle steps of the corresponding algorithms. It can cause some rounding error.

The aim of the proposed fuzzy TOPSIS is to obtain the organizational resilience factor that has the highest degree of satisfaction for all of the relevant business processes. The relative importance of business processes and the relative importance of organizational resilience factors with respect to each business process are modeled by triangular fuzzy numbers.

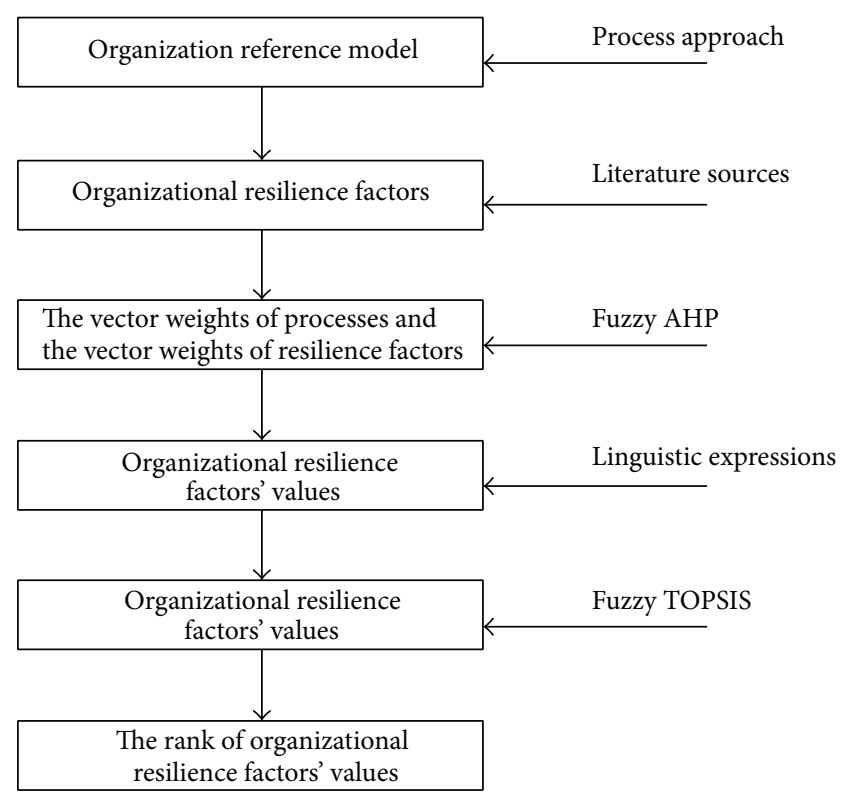

FIGURE 1: The evaluation procedure.

The vector of criteria weights of the business processes and organizational resilience factors with respect to each business process is given by using fuzzy AHP. The organizational resilience factor values are modeled by trapezoidal fuzzy numbers. First, we convert the decision matrix into a fuzzy decision matrix with respect to organizational resilience type. The weighted fuzzy decision matrix is constructed. The elements of this matrix are calculated as multiplication of the fuzzy rating value of an organizational resilience factor and its weight with respect to each business process. Then, we define FPIS and FNIS as $(1,1,1,1)$ and $(0,0,0,0)$, respectively (by analogy with [25]). The distance value of each resilience factor from the FPIS and FNIS is calculated by the expression developed in [26]. Finally, a closeness coefficient is determined for each organizational resilience factor by using the procedure which is proposed in the conventional TOPSIS, to determine the ranking of all organizational resilience factors. The higher value of the closeness coefficient indicates that an organizational resilience factor is closer to FPIS and further from FNIS, simultaneously. The organizational resilience factors are ranked according to decreasing order closeness coefficient values.

\section{Evaluation Framework}

The evaluation of organizational resilience factors with respect to all business processes and their weights can be stated as a multicriteria optimization task. The evaluation procedure in this paper consists of five steps as summarized in Figure 1.

Step 1. In order to make the evaluation process effective, it is necessary to define an organization reference model which will introduce the organizational elements that are going to be the base for the resilience factors' assessment. 
According to [27] an organization may be presented as a network of interrelated processes that are focused towards achieving organizational goals. In this paper, the organization reference model is represented by an interrelated network of $n$ business processes. The number, nature, and scope of business processes may depend on many factors with different significance (e.g., the economy sector, company size, or types of business activities). The assessment of organizational resilience factors should be done on the level of each defined business process.

Step 2. The identification of the organizational resilience factors that are considered as the most important for the treated organization is a very complex task and it is not distinguished by international consensus or standard definition [6]. Scholars have defined organizational resilience factors for different types of organizations [28, 29]. In this paper, each of these factors should be assessed and managed on the level of business processes defined by the organization reference model.

Step 3. After constructing the organizational resilience factors' hierarchy under each business process and constructing the hierarchy of business processes which is done by management team, the weights of the organizational resilience factors under each business process and weights of business processes by using FAHP [23] need to be calculated. A management team at the organizational level consists of main manager or owner and representatives of each business process (business process owners) because they are familiar with the processes and their condition. An assumption can be made that the management team will make a decision by consensus.

Step 4. The values of the organizational resilience factors are assessed by a management team. Modeling of the used linguistic expressions is performed by using fuzzy set theory $[7,8]$.

Step 5. The conducting of the fuzzy TOPSIS method is needed to achieve the final rank of the organizational resilience factors.

\section{Modeling of Uncertainties}

It is assumed that uncertainties are described by predefined linguistic expressions. The number and type of linguistic expressions are defined by decision makers. According to Zimmermann [30], the fuzzy sets theory could be the most appropriate way for modeling linguistic expressions. Each linguistic expression can be represented by a fuzzy number which can be assigned to a membership function.

A fuzzy set is represented by its membership function for which the parameters are shape, granularity, and location on the universe of discourse. The membership function shape of a fuzzy set can be obtained based on one's experience, the subjective belief of decision makers, intuition and contextual knowledge about the concept modeled [31], and uncertainty available for the treated linguistic variables [32]. However, subjectivity in determining membership function has been considered as the weakest point in fuzzy sets theory [33]. In the literature, the triangular and trapezoidal fuzzy numbers are commonly used for the modeling of different types of uncertainties which offer a good compromise between descriptive power and computational simplicity. Fuzzy sets of higher types and levels have not, as yet, played a significant role in applications of fuzzy sets theory [34]. In this paper, they are used. Granularity is defined as the number of fuzzy numbers assigned to theorganizational resilience factors and their values. It is suggested that only seven categories, at most, can be used [35]. In general, the domain of fuzzy sets can be defined on different measurement scales.

It can be mentioned that a different decision on the granularity and scale of domains used could lead to different results. Because of that, it cannot ensure the objectivity of the overall decision process.

4.1. Basic Definitions of Fuzzy Sets Theory. Some definitions of fuzzy sets presented in [36], using notations and specific terminology of decision theory, are reviewed.

Definition 1. Fuzzy set $\widetilde{A}$ is defined as a set of organized pairs:

$$
\widetilde{A}=\left\{x, \mu_{\widetilde{A}}(x) \mid x \in X, 0 \leq \mu_{\widetilde{A}}(x) \leq 1\right\},
$$

where fuzzy set $\widetilde{A}$ is defined on the universe set $X \in R$. In general, set $X$ can be either finite or infinite. $\mu_{\widetilde{A}}(x)$ is a membership function of fuzzy set $\widetilde{A}$.

Definition 2. A fuzzy number $\widetilde{A}$ is a convex normalized fuzzy set $\widetilde{A}$ of the real line $R$ such that: (1) It exists exactly one $x_{0} \in R$ with $\mu_{\widetilde{A}}\left(x_{0}\right)=1\left(x_{0}\right.$ is called the mean value of $\left.\widetilde{A}\right),(2) \mu_{\widetilde{A}}(x)$ is piecewise continuous.

Definition 3. Fuzzy number $\widetilde{A}$ on $R$ is to be a trapezoidal fuzzy number (TrFN) if its membership function $\mu_{\widetilde{A}}(x)$ : $R \rightarrow[0,1]$ is equal to

$$
\mu_{\widetilde{A}}(x)=\left\{\begin{array}{cc}
\frac{x-a}{b-a} & x \in[a, b] \\
1 & x \in[b, c] \\
\frac{d-x}{d-c} & x \in[c, d]
\end{array}\right\},
$$

where $a \leq b \leq c \leq d, a$ and $d$ stand for the lower and upper value of the support of $X$, respectively, and $c$ and $\mathrm{d}$ for the modal values. The trapezoidal fuzzy number can be denoted by $(a, b, c, d)$. The support of $X$ is the set of elements $\{x \in R \mid a<x<d\}$. When $a=b=c=d$, it is a nonfuzzy number by convention. 
If $b=c$ then $\widetilde{A}$ is called a triangular fuzzy number (TFN), denoted by $\widetilde{A}=(a, m, d)$, where $m=b=c$. Obviously, the membership function of the TFN is

$$
\mu_{\widetilde{A}}(x)=\left\{\begin{array}{cc}
\frac{x-a}{m-a} & x \in[a, m] \\
\frac{d-x}{d-m} & x \in[m, d] \\
0 & \text { otherwise }
\end{array}\right\} .
$$

Therefore, a triangular fuzzy number is a special case of the trapezoidal fuzzy number.

Definition 4. The trapezoidal fuzzy number can be denoted as $\widetilde{A}=(a, b, c, d)$, where $b$ and $c$ are modal values, and $a$ and $d$ denote the lower and upper bounds, respectively for nonzero values of $\mu_{\bar{A}}(x)$.

Definition 5. A matrix $\widetilde{M}$ is called a fuzzy matrix if at least one element in $\widetilde{M}$ is a fuzzy number [37].

Definition 6. If two fuzzy numbers $\widetilde{A}, B \in \widetilde{F}(R)$ with $\mu_{\widetilde{A}}(x)$ and $\mu_{\widetilde{B}}(x)$ continuous membership functions, then by application of the extension principle for the binary operation $*: R \otimes R \rightarrow R$, the membership function of the fuzzy number $\widetilde{A} * \widetilde{B}$ is given by $\mu_{\widetilde{A} * \widetilde{B}}(z)=\sup \min _{z=x * y}\left(\mu_{\widetilde{A}}(x), \mu_{\widetilde{B}}(y)\right)$.

Definition 7. The distance between two fuzzy numbers $\widetilde{A}$ and $\widetilde{B}$ which is denoted by $d_{p, q}(\cdot, \cdot)$ is a nonnegative function [26]. The analytic properties of $d_{p, q}(\cdot, \cdot)$ depend on the first parameter $p, 1<p<\infty$. The second parameter $q$ of $d_{p, q}(\cdot, \cdot)$ characterizes the subjective weighted value attributed to the end points of the support of the fuzzy numbers. If there is no reason for distinguishing any side of the fuzzy numbers, $d_{2,(1 / 2)}(\cdot, \cdot)$ is recommended.

By considering two trapezoidal fuzzy numbers $\widetilde{A}=$ $\left(a_{1}, a_{2}, a_{3}, a_{4}\right)$ and $\widetilde{B}=\left(b_{1}, b_{2}, b_{3}, b_{4}\right)$, the distance is calculated as

$$
\begin{aligned}
& d_{2,(1 / 2)}(\cdot, \cdot) \\
& \quad=\sqrt{\frac{1}{6} \cdot\left[\sum_{n=1}^{4}\left(b_{n}-a_{n}\right)^{2}+\sum_{n=\{1,3\}}\left(b_{n}-a_{n}\right) \cdot\left(b_{n+1}-a_{n+1}\right)\right]} .
\end{aligned}
$$

4.2. Modeling of the Relative Importance of Organizational Resilience Factors and the Relative Importance of Business Processes. It can be assumed that all the organizational resilience factors are not usually of the same relative importance for the considered business processes and that the business processes of the considered enterprise have different relative importance. Also, they can be considered as unchangeable during the considered period of time (one year). The relative importance of the organizational resilience factors can be changed if strategic management defines a new business strategy. The relative importance of business processes is determined according to literature data and the results of good practice. It may be assumed that the relative importance of business processes is unchangeable during a period of time.

The authors of this paper think that the judgement of each pair of treated variables best suits the nature of humandecision (by analogy with the AHP method). The use of a discrete scale of AHP is simple and easy, but it is not sufficient to take into account the uncertainty associated with the mapping of one's perception to a number [38]. It can be assumed that decision makers make the decision by consensus. The fuzzy rating of a management team is described by one of the five defined linguistic expressions.

On the other hand, the fuzzy pairwise comparison matrices are constructed of the relative importance of (1) the organizational resilience factors under each business process and (2) the business processes. The elements of these fuzzy pairwise comparison matrices are triangular fuzzy numbers. The relative importance of each pair of organizational resilience factors under each business process is described by triangular fuzzy numbers $\widetilde{W}_{i i^{\prime}}^{p}=\left(x ; l_{i i^{\prime}}^{p}, m_{i i^{\prime}}^{p}, u_{i i^{\prime}}^{p}\right)$. In similar way the relative importance of each pair of business processes may be considered and presented by triangular fuzzy number $\widetilde{W}_{p p^{\prime}}=\left(x ; l_{p p^{\prime}}, m_{p p^{\prime}}, u_{p p^{\prime}}\right), i, i^{\prime}=1, \ldots, I ; p, p^{\prime}=1, \ldots, p ;$ $i \neq i^{\prime} ; p \neq p^{\prime}$.

The lower and upper bounds $l_{i i^{\prime}}^{p}, l_{p p^{\prime}}, u_{i i^{\prime}}^{p}$ and $u_{p p^{\prime}}$ and modal values $m_{i i^{\prime}}^{p}$ and $m_{p p^{\prime}}$, belong to the interval [1-5]. Value 1 indicates that the relative importance of organizational resilience factor $i$ compared to the factor $i^{\prime}$ and process $p$ compared to the process $p^{\prime}$ is equal. Value 5 indicates that factor $i$ and process $p$ are extremely more important than indicator $i^{\prime}$ and process $p^{\prime}$, respectively.

If the strong relative importance of organizational resilience factor $i^{\prime}$ over organizational resilience factor $i$ under each considered business process holds, then the pairwise comparison scale can be represented by the fuzzy number $\widetilde{W}_{i i^{\prime}}^{p}=\left(\widetilde{W}_{i i^{\prime}}^{p}\right)^{-1}=\left(\left(1 / u_{i i^{\prime}}^{p}\right),\left(1 / m_{i i^{\prime}}^{p}\right),\left(1 / l_{i i^{\prime}}^{p}\right)\right)$. In a similar way, it may be presented as $\widetilde{W}_{p p^{\prime}}=\left(\widetilde{W}_{p p^{\prime}}\right)^{-1}=$ $\left(\left(1 / u_{p p^{\prime}}\right),\left(1 / m_{p p^{\prime}}\right),\left(1 / l_{p p^{\prime}}\right)\right)$.

If $i=i^{\prime}$ and $p=p^{\prime}\left(i, i^{\prime}=1, \ldots, I\right.$ and $\left.p, p^{\prime}=1, \ldots, P\right)$ then the relative importance of organizational resilience factor $i$ over organizational resilience factor $i^{\prime}$ under business process $p$ and the relative importance of business process $p$ over business process $p^{\prime}$ are represented by a single point 1 which is a triangular fuzzy number $(1,1,1)$.

In this paper, the management team uses five linguistic expressions which are modeled by triangular fuzzy numbers which are given in the following way:

$$
\begin{aligned}
& \text { very low importance- }-\widetilde{R}_{1}=(x ; 1,1,3.5), \\
& \text { low importance }-\widetilde{R}_{2}=(x ; 1,1,5), \\
& \text { moderate importance- }-\widetilde{R}_{3}=(x ; 1,3,5), \\
& \text { high importance- }-\widetilde{R}_{4}=(x ; 1,5,5), \\
& \text { very high importance }-\widetilde{R}_{5}=(x ; 2.5,5,5) .
\end{aligned}
$$

The vector of weights of the identified organizational resilience factors under each business process and the vector 
of weights of the considered business processes are calculated by applying the concept of extent analysis [23]. The normalized vector of weights of the organizational resilience factors under business process $p$ is denoted by $W_{i p}=$ $\left(w_{i p}, \ldots, w_{i p}, \ldots, w_{I p}\right), i=1, \ldots, I$ and $p=1, \ldots, P$. The normalized vector of weights of the business processws is denoted by $W_{p}=\left(w_{1}, \ldots, w_{p}, \ldots, w_{p}\right), p=1, \ldots, P$.

$W_{i p}$ and $W_{p}$ are nonfuzzy numbers and this gives the priority weights of one organizational resilience factor under business process $p, p=1, \ldots, P$, respectively, and one business process over the other organizational resilience factors under business process $p$ in relation to other business processes.

4.3. Modeling of Organizational Resilience Factor Values. The organizational resilience factor values at the level of business processes are not precisely measurable variables. Their values are determined by assessment of the management team by consensus. The management team uses appropriate linguistic expressions for describing the organizational resilience factor values. These linguistic expressions are modeled by triangular fuzzy numbers $\widetilde{v}_{i p}=\left(x ; a_{i p}, b_{i p}, c_{i p}, d_{i p}\right)$ with the lower and upper bounds $a_{i p}$ and $d_{i p}$ and modal values $b_{i p}$ and $c_{i p}$, respectively.

For the problem considered in this paper, the five linguistic expressions are proposed, which are modeled by trapezoidal fuzzy numbers as follows:

$$
\begin{aligned}
& \text { very low value }-\widetilde{v}_{1}=(y ; 0,0,0.1,0.2) \\
& \text { low value }-\widetilde{v}_{2}=(y ; 0,0.1,0.2,0.3), \\
& \text { medium low value }-\widetilde{v}_{3}=(y ; 0.15,0.3,0.4,0.55), \\
& \text { medium value }-\widetilde{v}_{4}=(y ; 0.25,0.4,0.5,0.65), \\
& \text { medium high value }-\widetilde{v}_{5}=(y ; 0.55,0.7,0.8,0.95), \\
& \text { high value }-\widetilde{v}_{6}=(y ; 0.7,0.8,0.9,1), \\
& \text { very high value }-\widetilde{v}_{7}=(y ; 0.8,0.9,1,1) .
\end{aligned}
$$

\section{The Proposed Fuzzy TOPSIS Algorithm}

The algorithm of the proposed novel fuzzy TOPSIS is formally given as follows.

Step 1. Construct the fuzzy pairwise comparison matrix of the relative importance of business processes, $\widetilde{W}_{p p^{\prime}}=$ $\left(x ; l_{p p^{\prime}}, m_{p p^{\prime}}, u_{p p^{\prime}}\right), p, p^{\prime}=1, \ldots, P, p \neq p^{\prime}$.

By using extent AHP analysis [23] the vector of weights, $W_{p}=\left[w_{p}\right]_{1 \times P}, p=1, \ldots, P$, is calculated.

Step 2. Construct the fuzzy pairwise comparison matrix of the relative importance of business processes $\widetilde{W}_{i i^{\prime}}^{p}=$ $\left(x ; l_{i i^{\prime}}^{p}, m_{i i^{\prime}}^{p}, u_{i i^{\prime}}^{p}\right), i, i^{\prime}=1, \ldots, I, i \neq i^{\prime}$.

By using extent AHP analysis [23] the vector of weights, $W_{i p}=\left[w_{i p}\right]_{1 x P}, p=1, \ldots, P, i=1, \ldots, I$ is calculated.
Step 3. The weighted normalized fuzzy decision matrix denoted by $\widetilde{F}$ is shown as

$$
\widetilde{F}=\left[\tilde{f}_{i p}\right]_{I x P},
$$

where $\tilde{f}_{i p}=w_{i p} \cdot \widetilde{v}_{i p}, i=1, \ldots, I, p=1, \ldots, P$.

Step 4. Define FPIS and FNIS according to [39] so that

$$
\widetilde{v}_{p}^{+}=(1,1,1,1), \quad \widetilde{v}_{p}^{-}=(0,0,0,0) .
$$

Step 5. Calculate the separation measures for each organizational resilience factor i from the FPIS, $d_{i}^{+}$and FNIS, $d_{i}^{-}$using the following expressions:

$$
\begin{array}{r}
d_{i}^{+}=\sum_{p=1}^{P} w_{p} \cdot\left(\widetilde{v}_{p}^{-}, \widetilde{f}_{i p}\right), \quad d_{i}^{-}=\sum_{p=1}^{P} w_{p} \cdot\left(\widetilde{v}_{p}^{-}, \widetilde{f}_{i p}\right), \\
i=1, \ldots, I .
\end{array}
$$

Step 6. The closeness coefficient based on the distance measure can be calculated as follows:

$$
c_{i}=\frac{d_{i}^{-}}{d_{i}^{-}+d_{i}^{+}} .
$$

Step 7. Rank preference order. The values $c_{i}, i=1, \ldots, I$, should be sorted in decreasing order. A rank organizational resilience factor corresponds to the rank of closeness coefficients.

\section{A Real Case Study}

The proposed FTOPSIS is tested on real data given from one SME of the process industry in the Republic of Serbia. The process industry has a very significant role, overall, in SMEs of the Serbian economy (Republic Statistical Office of Serbia, 2010). The analysed organization is certified according to ISO 9001 Standard which is crucial for the reference organization model. The identified business processes are management $(p=1)$, marketing and sales $(p=2)$, design and development $(p=3)$, purchase $(p=4)$, production $(p=5)$, and support processes $(p=6)$. The considered organizational resilience factors and their values are determined according to Aleksic [29]. These resilience factors are briefly described as follows:

(1) planning strategies: associated mostly with the process of management and strategy; they should be assessed in the manner of the deployed strategies for achieving resilience;

(2) the capability and capacity of internal resources: scoped to the internal processes which should be assessed in the manner of adequate management of the processing of resources and existing procedures in a time of crisis;

(3) internal situation monitoring and reporting: integrated with internal processes which should be assessed in the scope of an organizational information system and assets that are dedicated to organizational awareness; 
(4) human factors: associated with human resources that are scoped and assessed on the level of competencies and human motivation;

(5) quality: integrated in all business processes which should be assessed in the scope of the defined quality goals and their overall monitoring and measurement;

(6) external situation monitoring and reporting: associated mostly with external processes which should be assessed in the scope of information processing from outside of the organization through the information system within the organization;

(7) capability and capacity of external resources: integrated with external resources which should be analysed in the scope of adequate management of process resources that are introduced from outside the organization, such as power supply or electricity;

(8) design factor: integrated with the manufacturing process operations; it should be assessed in the scope of the processes' design as well as the industrial assets' design;

(9) detection potential: associated with possible deviations from process realization; its assessment should take into account the functioning of the defined procedures for detection of faults within the processes;

(10) emergency response: it is scoped to the response in a time of crisis; its assessment should take into account existing procedures for organizational response which should be implemented in a time of crisis;
(11) safety management system: it is associated with safety of employees and the safety of industrial assets; its assessment should take into account the functioning of defined procedures for safety of employees and the safety of industrial assets.

A fuzzy pairwise comparison matrix of the relative importance of business processes is

$$
\left[\begin{array}{cccccc}
(1,1,1) & \widetilde{R}_{4} & \widetilde{R}_{5} & \widetilde{R}_{4} & \widetilde{R}_{2} & \widetilde{R}_{4} \\
\frac{1}{\widetilde{R}_{4}} & (1,1,1) & \widetilde{R}_{1} & \frac{1}{\widetilde{R}_{1}} & \frac{1}{\widetilde{R}_{3}} & \frac{1}{\widetilde{R}_{1}} \\
\frac{1}{\widetilde{R}_{5}} & \frac{1}{\widetilde{R}_{1}} & (1,1,1) & \frac{1}{\widetilde{R}_{2}} & \frac{1}{\widetilde{R}_{3}} & \frac{1}{\widetilde{R}_{4}} \\
\frac{1}{\widetilde{R}_{4}} & \widetilde{R}_{1} & \widetilde{R}_{2} & (1,1,1) & \frac{1}{\widetilde{R}_{2}} & \widetilde{R}_{2} \\
\frac{1}{\widetilde{R}_{2}} & \widetilde{R}_{3} & \widetilde{R}_{3} & \widetilde{R}_{2} & (1,1,1) & \widetilde{R}_{4} \\
\frac{1}{\widetilde{R}_{4}} & \widetilde{R}_{1} & \widetilde{R}_{4} & \frac{1}{\widetilde{R}_{2}} & \frac{1}{\widetilde{R}_{4}} & (1,1,1)
\end{array}\right] .
$$

The fuzzy pairwise comparison matrix of the relative importance of the organizational resilience factors with respect to process management $(p=1)$ is

$$
\left[\begin{array}{ccccccccccc}
1,1,1 & \frac{1}{\widetilde{R}_{2}} & \frac{1}{\widetilde{R}_{2}} & \frac{1}{\widetilde{R}_{3}} & \frac{1}{\widetilde{R}_{4}} & \frac{1}{\widetilde{R}_{1}} & \frac{1}{\widetilde{R}_{3}} & \frac{1}{\widetilde{R}_{2}} & \frac{1}{\widetilde{R}_{5}} & \frac{1}{\widetilde{R}_{2}} & \frac{1}{\widetilde{R}_{3}} \\
\widetilde{R}_{2} & 1,1,1 & \frac{1}{\widetilde{R}_{1}} & \frac{1}{\widetilde{R}_{2}} & \frac{1}{\widetilde{R}_{5}} & \frac{1}{\widetilde{R}_{1}} & \frac{1}{\widetilde{R}_{2}} & \frac{1}{\widetilde{R}_{1}} & \frac{1}{\widetilde{R}_{4}} & \frac{1}{\widetilde{R}_{1}} & \frac{1}{\widetilde{R}_{3}} \\
\widetilde{R}_{2} & \widetilde{R}_{1} & 1,1,1 & 1,1,1 & \frac{1}{\widetilde{R}_{4}} & \widetilde{R}_{2} & \frac{1}{\widetilde{R}_{2}} & \frac{1}{\widetilde{R}_{1}} & \frac{1}{\widetilde{R}_{4}} & \widetilde{R}_{2} & \frac{1}{\widetilde{R}_{3}} \\
\widetilde{R}_{3} & \widetilde{R}_{2} & 1,1,1 & 1,1,1 & \frac{1}{\widetilde{R}_{3}} & 1,1,1 & \frac{1}{\widetilde{R}_{2}} & \frac{1}{\widetilde{R}_{2}} & \frac{1}{\widetilde{R}_{3}} & 1,1,1 & \frac{1}{\widetilde{R}_{2}} \\
\widetilde{R}_{4} & \widetilde{R}_{5} & \widetilde{R}_{4} & \widetilde{R}_{3} & 1,1,1 & \widetilde{R}_{3} & \widetilde{R}_{1} & \widetilde{R}_{3} & 1,1,1 & \widetilde{R}_{3} & \frac{1}{\widetilde{R}_{1}} \\
\widetilde{R}_{1} & \widetilde{R}_{1} & \frac{1}{\widetilde{R}_{2}} & 1,1,1 & \frac{1}{\widetilde{R}_{3}} & 1,1,1 & \frac{1}{\widetilde{R}_{2}} & 1,1,1 & \frac{1}{\widetilde{R}_{3}} & 1,1,1 & \frac{1}{\widetilde{R}_{2}} \\
\widetilde{R}_{3} & \widetilde{R}_{2} & \widetilde{R}_{2} & \widetilde{R}_{2} & \frac{1}{\widetilde{R}_{1}} & \widetilde{R}_{2} & 1,1,1 & \widetilde{R}_{2} & \frac{1}{\widetilde{R}_{2}} & \widetilde{R}_{2} & \frac{1}{\widetilde{R}_{2}} \\
\widetilde{R}_{2} & \widetilde{R}_{1} & \widetilde{R}_{1} & \widetilde{R}_{2} & \frac{1}{\widetilde{R}_{3}} & 1,1,1 & \frac{1}{\widetilde{R}_{2}} & 1,1,1 & \frac{1}{\widetilde{R}_{3}} & \widetilde{R}_{2} & \frac{1}{\widetilde{R}_{3}} \\
\widetilde{R}_{5} & \widetilde{R}_{4} & \widetilde{R}_{4} & \widetilde{R}_{3} & 1,1,1 & \widetilde{R}_{3} & \widetilde{R}_{2} & \widetilde{R}_{3} & 1,1,1 & \widetilde{R}_{5} & \widetilde{R}_{2} \\
\widetilde{R}_{2} & \widetilde{R}_{1} & \frac{1}{\widetilde{R}_{2}} & 1,1,1 & \frac{1}{\widetilde{R}_{3}} & 1,1,1 & \frac{1}{\widetilde{R}_{2}} & \frac{1}{\widetilde{R}_{2}} & \frac{1}{\widetilde{R}_{5}} & 1,1,1 & \frac{1}{\widetilde{R}_{4}} \\
\widetilde{R}_{3} & \widetilde{R}_{2} & \widetilde{R}_{1} & \widetilde{R}_{2} & \widetilde{R}_{2} & \widetilde{R}_{3} & \frac{1}{\widetilde{R}_{2}} & \widetilde{R}_{4} & 1,1,1
\end{array}\right] .
$$


The fuzzy pairwise comparison matrix of the relative importance of the organizational resilience factors with respect to process marketing and sales $(p=2)$ is

$$
\left[\begin{array}{ccccccccccc}
1,1,1 & 1,1,1 & \frac{1}{\widetilde{R}_{2}} & \frac{1}{\widetilde{R}_{1}} & \frac{1}{\widetilde{R}_{5}} & \frac{1}{\widetilde{R}_{3}} & \frac{1}{\widetilde{R}_{4}} & \frac{1}{\widetilde{R}_{2}} & \frac{1}{\widetilde{R}_{3}} & 1,1,1 & \frac{1}{\widetilde{R}_{1}} \\
1,1,1 & 1,1,1 & \frac{1}{\widetilde{R}_{2}} & \frac{1}{\widetilde{R}_{1}} & \frac{1}{\widetilde{R}_{3}} & \frac{1}{\widetilde{R}_{2}} & \frac{1}{\widetilde{R}_{2}} & \frac{1}{\widetilde{R}_{2}} & \frac{1}{\widetilde{R}_{3}} & \frac{1}{\widetilde{R}_{1}} & \frac{1}{\widetilde{R}_{1}} \\
\widetilde{R}_{2} & \widetilde{R}_{2} & 1,1,1 & \frac{1}{\widetilde{R}_{1}} & \frac{1}{\widetilde{R}_{3}} & \widetilde{R}_{1} & \widetilde{R}_{1} & \widetilde{R}_{2} & \frac{1}{\widetilde{R}_{3}} & \widetilde{R}_{1} & \frac{1}{\widetilde{R}_{1}} \\
\widetilde{R}_{1} & \widetilde{R}_{1} & \widetilde{R}_{1} & 1,1,1 & \frac{1}{\widetilde{R}_{2}} & \widetilde{R}_{1} & \frac{1}{\widetilde{R}_{3}} & \frac{1}{\widetilde{R}_{1}} & \frac{1}{\widetilde{R}_{2}} & \widetilde{R}_{2} & \frac{1}{\widetilde{R}_{1}} \\
\widetilde{R}_{5} & \widetilde{R}_{3} & \widetilde{R}_{3} & \widetilde{R}_{2} & 1,1,1 & \widetilde{R}_{1} & \widetilde{R}_{1} & \widetilde{R}_{1} & \frac{1}{\widetilde{R}_{2}} & \widetilde{R}_{3} & \frac{1}{\widetilde{R}_{3}} \\
\widetilde{R}_{3} & \widetilde{R}_{2} & \frac{1}{\widetilde{R}_{1}} & \frac{1}{\widetilde{R}_{1}} & \frac{1}{\widetilde{R}_{1}} & 1,1,1 & \frac{1}{\widetilde{R}_{1}} & \widetilde{R}_{1} & \frac{1}{\widetilde{R}_{1}} & \widetilde{R}_{2} & \frac{1}{\widetilde{R}_{1}} \\
\widetilde{R}_{4} & \widetilde{R}_{2} & \frac{1}{\widetilde{R}_{1}} & \widetilde{R}_{3} & \frac{1}{\widetilde{R}_{1}} & \widetilde{R}_{1} & 1,1,1 & \widetilde{R}_{2} & \frac{1}{\widetilde{R}_{1}} & \widetilde{R}_{2} & \frac{1}{\widetilde{R}_{2}} \\
\widetilde{R}_{2} & \widetilde{R}_{2} & \frac{1}{\widetilde{R}_{2}} & \widetilde{R}_{1} & \frac{1}{\widetilde{R}_{1}} & \frac{1}{\widetilde{R}_{1}} & \frac{1}{\widetilde{R}_{2}} & 1,1,1 & \frac{1}{\widetilde{R}_{2}} & \widetilde{R}_{1} & \frac{1}{\widetilde{R}_{2}} \\
\widetilde{R}_{3} & \widetilde{R}_{3} & \widetilde{R}_{3} & \widetilde{R}_{2} & \widetilde{R}_{2} & \widetilde{R}_{1} & \widetilde{R}_{1} & \widetilde{R}_{2} & 1,1,1 & \widetilde{R}_{3} & 1,1,1 \\
1,1,1 & \widetilde{R}_{1} & \frac{1}{\widetilde{R}_{1}} & \frac{1}{\widetilde{R}_{2}} & \frac{1}{\widetilde{R}_{3}} & \frac{1}{\widetilde{R}_{2}} & \frac{1}{\widetilde{R}_{2}} & \frac{1}{\widetilde{R}_{1}} & \frac{1}{\widetilde{R}_{3}} & 1,1,1 & \frac{1}{\widetilde{R}_{1}} \\
\widetilde{R}_{1} & \widetilde{R}_{1} & \widetilde{R}_{1} & \widetilde{R}_{1} & \widetilde{R}_{3} & \widetilde{R}_{1} & \widetilde{R}_{2} & \widetilde{R}_{2} & 1,1,1 & \widetilde{R}_{1} & 1,1,1
\end{array}\right] .
$$

The fuzzy pairwise comparison matrix of the relative importance of the organizational resilience factors with respect to process design and development $(p=3)$ is

$$
\left[\begin{array}{ccccccccccc}
1,1,1 & \frac{1}{\widetilde{R}_{2}} & \widetilde{R}_{1} & \frac{1}{\widetilde{R}_{2}} & \frac{1}{\widetilde{R}_{3}} & \frac{1}{\widetilde{R}_{5}} & \frac{1}{\widetilde{R}_{3}} & \frac{1}{\widetilde{R}_{2}} & \frac{1}{\widetilde{R}_{4}} & \widetilde{R}_{1} & \frac{1}{\widetilde{R}_{1}} \\
\widetilde{R}_{2} & 1,1,1 & \frac{1}{\widetilde{R}_{2}} & \frac{1}{\widetilde{R}_{2}} & \frac{1}{\widetilde{R}_{2}} & \frac{1}{\widetilde{R}_{3}} & \frac{1}{\widetilde{R}_{1}} & \frac{1}{\widetilde{R}_{1}} & \frac{1}{\widetilde{R}_{3}} & \frac{1}{\widetilde{R}_{1}} & \frac{1}{\widetilde{R}_{2}} \\
\frac{1}{\widetilde{R}_{1}} & \widetilde{R}_{2} & 1,1,1 & \frac{1}{\widetilde{R}_{2}} & \frac{1}{\widetilde{R}_{4}} & \frac{1}{\widetilde{R}_{3}} & \frac{1}{\widetilde{R}_{2}} & \widetilde{R}_{1} & \frac{1}{\widetilde{R}_{5}} & \frac{1}{\widetilde{R}_{1}} & \frac{1}{\widetilde{R}_{2}} \\
\widetilde{R}_{2} & \widetilde{R}_{2} & \widetilde{R}_{2} & 1,1,1 & \frac{1}{\widetilde{R}_{1}} & \frac{1}{\widetilde{R}_{3}} & \frac{1}{\widetilde{R}_{1}} & \widetilde{R}_{2} & 1,1,1 & \widetilde{R}_{1} & \frac{1}{\widetilde{R}_{3}} \\
\widetilde{R}_{3} & \widetilde{R}_{2} & \widetilde{R}_{4} & \widetilde{R}_{1} & 1,1,1 & \frac{1}{\widetilde{R}_{1}} & \widetilde{R}_{2} & \widetilde{R}_{2} & 1,1,1 & \widetilde{R}_{4} & \widetilde{R}_{2} \\
\widetilde{R}_{5} & \widetilde{R}_{3} & \widetilde{R}_{3} & \widetilde{R}_{3} & \widetilde{R}_{1} & 1,1,1 & \widetilde{R}_{1} & \widetilde{R}_{1} & \frac{1}{\widetilde{R}_{2}} & \widetilde{R}_{4} & \frac{1}{\widetilde{R}_{1}} \\
\widetilde{R}_{3} & \widetilde{R}_{1} & \widetilde{R}_{2} & \widetilde{R}_{1} & \frac{1}{\widetilde{R}_{2}} & \frac{1}{\widetilde{R}_{1}} & 1,1,1 & \widetilde{R}_{1} & \frac{1}{\widetilde{R}_{3}} & \widetilde{R}_{1} & \frac{1}{\widetilde{R}_{1}} \\
\widetilde{R}_{2} & \widetilde{R}_{1} & \frac{1}{\widetilde{R}_{1}} & \frac{1}{\widetilde{R}_{2}} & \frac{1}{\widetilde{R}_{2}} & \frac{1}{\widetilde{R}_{1}} & \frac{1}{\widetilde{R}_{1}} & 1,1,1 & \frac{1}{\widetilde{R}_{3}} & \widetilde{R}_{1} & \frac{1}{\widetilde{R}_{1}} \\
\widetilde{R}_{4} & \widetilde{R}_{3} & \widetilde{R}_{5} & 1,1,1 & 1,1,1 & \widetilde{R}_{2} & \widetilde{R}_{3} & \widetilde{R}_{3} & 1,1,1 & \widetilde{R}_{5} & \widetilde{R}_{1} \\
\frac{1}{\widetilde{R}_{1}} & \widetilde{R}_{1} & \widetilde{R}_{1} & \frac{1}{\widetilde{R}_{1}} & \frac{1}{\widetilde{R}_{4}} & \frac{1}{\widetilde{R}_{4}} & \frac{1}{\widetilde{R}_{1}} & \frac{1}{\widetilde{R}_{1}} & \frac{1}{\widetilde{R}_{5}} & 1,1,1 & \frac{1}{\widetilde{R}_{1}} \\
\widetilde{R}_{1} & \widetilde{R}_{2} & \widetilde{R}_{2} & \widetilde{R}_{3} & \frac{1}{\widetilde{R}_{2}} & \widetilde{R}_{1} & \widetilde{R}_{1} & \widetilde{R}_{1} & \frac{1}{\widetilde{R}_{1}} & \widetilde{R}_{1} & 1,1,1
\end{array}\right] .
$$


The fuzzy pairwise comparison matrix of the relative importance of the organizational resilience factors with respect to process purchase $(p=4)$ is

$$
\left[\begin{array}{ccccccccccc}
1,1,1 & \frac{1}{\widetilde{R}_{3}} & \frac{1}{\widetilde{R}_{1}} & \frac{1}{\widetilde{R}_{1}} & \frac{1}{\widetilde{R}_{2}} & \frac{1}{\widetilde{R}_{3}} & \frac{1}{\widetilde{R}_{2}} & \frac{1}{\widetilde{R}_{2}} & \frac{1}{\widetilde{R}_{3}} & 1,1,1 & \frac{1}{\widetilde{R}_{1}} \\
\widetilde{R}_{3} & 1,1,1 & \widetilde{R}_{1} & \widetilde{R}_{2} & \frac{1}{\widetilde{R}_{1}} & 1,1,1 & \widetilde{R}_{2} & \widetilde{R}_{2} & \frac{1}{\widetilde{R}_{2}} & \widetilde{R}_{1} & \frac{1}{\widetilde{R}_{2}} \\
\widetilde{R}_{1} & \frac{1}{\widetilde{R}_{1}} & 1,1,1 & \frac{1}{\widetilde{R}_{1}} & \frac{1}{\widetilde{R}_{1}} & \widetilde{R}_{2} & \widetilde{R}_{1} & \frac{1}{\widetilde{R}_{1}} & \frac{1}{\widetilde{R}_{1}} & \widetilde{R}_{1} & 1,1,1 \\
\widetilde{R}_{1} & \frac{1}{\widetilde{R}_{2}} & \widetilde{R}_{1} & 1,1,1 & \widetilde{R}_{1} & \widetilde{R}_{2} & \frac{1}{\widetilde{R}_{2}} & \frac{1}{\widetilde{R}_{1}} & \frac{1}{\widetilde{R}_{3}} & \widetilde{R}_{1} & \frac{1}{\widetilde{R}_{2}} \\
\widetilde{R}_{2} & \widetilde{R}_{1} & \widetilde{R}_{1} & \frac{1}{\widetilde{R}_{1}} & 1,1,1 & \widetilde{R}_{5} & \widetilde{R}_{1} & \widetilde{R}_{1} & \frac{1}{\widetilde{R}_{2}} & \widetilde{R}_{2} & \frac{1}{\widetilde{R}_{2}} \\
\widetilde{R}_{3} & 1,1,1 & \frac{1}{\widetilde{R}_{2}} & \frac{1}{\widetilde{R}_{2}} & \frac{1}{\widetilde{R}_{5}} & 1,1,1 & \frac{1}{\widetilde{R}_{3}} & \frac{1}{\widetilde{R}_{2}} & \frac{1}{\widetilde{R}_{3}} & \frac{1}{\widetilde{R}_{1}} & \frac{1}{\widetilde{R}_{2}} \\
\widetilde{R}_{3} & \frac{1}{\widetilde{R}_{2}} & \frac{1}{\widetilde{R}_{1}} & \widetilde{R}_{2} & \frac{1}{\widetilde{R}_{1}} & \widetilde{R}_{3} & 1,1,1 & \frac{1}{\widetilde{R}_{1}} & \frac{1}{\widetilde{R}_{2}} & \widetilde{R}_{2} & \frac{1}{\widetilde{R}_{1}} \\
\widetilde{R}_{2} & \frac{1}{\widetilde{R}_{2}} & \widetilde{R}_{1} & \widetilde{R}_{1} & \frac{1}{\widetilde{R}_{1}} & \widetilde{R}_{2} & \widetilde{R}_{1} & 1,1,1 & \frac{1}{\widetilde{R}_{2}} & \widetilde{R}_{2} & \frac{1}{\widetilde{R}_{1}} \\
\widetilde{R}_{3} & \widetilde{R}_{2} & \widetilde{R}_{1} & \widetilde{R}_{3} & \widetilde{R}_{2} & \widetilde{R}_{3} & \widetilde{R}_{2} & \widetilde{R}_{2} & 1,1,1 & \widetilde{R}_{2} & \widetilde{R}_{1} \\
1,1,1 & \frac{1}{\widetilde{R}_{1}} & \frac{1}{\widetilde{R}_{1}} & \frac{1}{\widetilde{R}_{1}} & \frac{1}{\widetilde{R}_{2}} & \widetilde{R}_{1} & \frac{1}{\widetilde{R}_{2}} & \frac{1}{\widetilde{R}_{2}} & \frac{1}{\widetilde{R}_{2}} & 1,1,1 & \frac{1}{\widetilde{R}_{1}} \\
\widetilde{R}_{1} & \widetilde{R}_{2} & 1,1,1 & \widetilde{R}_{2} & \widetilde{R}_{2} & \widetilde{R}_{2} & \widetilde{R}_{1} & \widetilde{R}_{1} & \frac{1}{\widetilde{R}_{1}} & \widetilde{R}_{1} & 1,1,1
\end{array}\right]
$$

The fuzzy pairwise comparison matrix of the relative importance of the organizational resilience factors with respect to process production $(p=5)$ is

$$
\left[\begin{array}{ccccccccccc}
1,1,1 & 1,1,1 & \frac{1}{\widetilde{R}_{1}} & \frac{1}{\widetilde{R}_{1}} & \frac{1}{\widetilde{R}_{3}} & \widetilde{R}_{2} & \frac{1}{\widetilde{R}_{2}} & \frac{1}{\widetilde{R}_{2}} & \frac{1}{\widetilde{R}_{4}} & \frac{1}{\widetilde{R}_{1}} & \frac{1}{\widetilde{R}_{2}} \\
1,1,1 & 1,1,1 & \frac{1}{\widetilde{R}_{2}} & \widetilde{R}_{1} & \frac{1}{\widetilde{R}_{2}} & 1,1,1 & \frac{1}{\widetilde{R}_{3}} & \frac{1}{\widetilde{R}_{3}} & \frac{1}{\widetilde{R}_{1}} & \frac{1}{\widetilde{R}_{2}} & \frac{1}{\widetilde{R}_{2}} \\
\widetilde{R}_{1} & \widetilde{R}_{2} & 1,1,1 & \widetilde{R}_{1} & \frac{1}{\widetilde{R}_{2}} & \widetilde{R}_{1} & \frac{1}{\widetilde{R}_{1}} & \frac{1}{\widetilde{R}_{2}} & \frac{1}{\widetilde{R}_{2}} & \widetilde{R}_{1} & \frac{1}{\widetilde{R}_{1}} \\
\widetilde{R}_{1} & \frac{1}{\widetilde{R}_{1}} & \frac{1}{\widetilde{R}_{1}} & 1,1,1 & \frac{1}{\widetilde{R}_{1}} & \widetilde{R}_{2} & \frac{1}{\widetilde{R}_{1}} & \frac{1}{\widetilde{R}_{2}} & \frac{1}{\widetilde{R}_{1}} & \frac{1}{\widetilde{R}_{1}} & \frac{1}{\widetilde{R}_{1}} \\
\widetilde{R}_{3} & \widetilde{R}_{2} & \widetilde{R}_{2} & \widetilde{R}_{1} & 1,1,1 & \widetilde{R}_{1} & \widetilde{R}_{2} & \widetilde{R}_{1} & \frac{1}{\widetilde{R}_{1}} & \widetilde{R}_{2} & \frac{1}{\widetilde{R}_{1}} \\
\frac{1}{\widetilde{R}_{2}} & 1,1,1 & \frac{1}{\widetilde{R}_{1}} & \frac{1}{\widetilde{R}_{2}} & \frac{1}{\widetilde{R}_{1}} & 1,1,1 & \frac{1}{\widetilde{R}_{3}} & \frac{1}{\widetilde{R}_{1}} & \frac{1}{\widetilde{R}_{4}} & \frac{1}{\widetilde{R}_{3}} & \frac{1}{\widetilde{R}_{3}} \\
\widetilde{R}_{2} & \widetilde{R}_{3} & \widetilde{R}_{1} & \widetilde{R}_{1} & \frac{1}{\widetilde{R}_{2}} & \widetilde{R}_{3} & 1,1,1 & \widetilde{R}_{1} & \frac{1}{\widetilde{R}_{3}} & \widetilde{R}_{2} & \frac{1}{\widetilde{R}_{1}} \\
\widetilde{R}_{2} & \widetilde{R}_{3} & \widetilde{R}_{2} & \widetilde{R}_{2} & \frac{1}{\widetilde{R}_{1}} & \widetilde{R}_{1} & \frac{1}{\widetilde{R}_{1}} & 1,1,1 & \frac{1}{\widetilde{R}_{2}} & \widetilde{R}_{1} & \frac{1}{\widetilde{R}_{1}} \\
\widetilde{R}_{4} & \widetilde{R}_{1} & \widetilde{R}_{2} & \widetilde{R}_{1} & \widetilde{R}_{1} & \widetilde{R}_{4} & \widetilde{R}_{3} & \widetilde{R}_{2} & 1,1,1 & \widetilde{R}_{2} & 1,1,1 \\
\widetilde{R}_{1} & \widetilde{R}_{2} & \frac{1}{\widetilde{R}_{1}} & \widetilde{R}_{1} & \frac{1}{\widetilde{R}_{2}} & \widetilde{R}_{3} & \frac{1}{\widetilde{R}_{2}} & \frac{1}{\widetilde{R}_{1}} & \frac{1}{\widetilde{R}_{2}} & 1,1,1 & \frac{1}{\widetilde{R}_{1}} \\
\widetilde{R}_{2} & \widetilde{R}_{2} & \widetilde{R}_{1} & \widetilde{R}_{1} & \widetilde{R}_{1} & \widetilde{R}_{3} & \widetilde{R}_{1} & \widetilde{R}_{1} & 1,1,1 & \widetilde{R}_{1} & 1,1,1
\end{array}\right]
$$


The fuzzy pairwise comparison matrix of the relative importance of the organizational resilience factors with respect to support processes $(p=6)$ is

$$
\left[\begin{array}{ccccccccccc}
1,1,1 & \widetilde{R}_{1} & 1,1,1 & \frac{1}{\widetilde{R}_{1}} & \frac{1}{\widetilde{R}_{2}} & 1,1,1 & \frac{1}{\widetilde{R}_{2}} & \frac{1}{\widetilde{R}_{1}} & \frac{1}{\widetilde{R}_{3}} & \frac{1}{\widetilde{R}_{1}} & \frac{1}{\widetilde{R}_{1}} \\
\frac{1}{\widetilde{R}_{1}} & 1,1,1 & \widetilde{R}_{2} & \frac{1}{\widetilde{R}_{1}} & \frac{1}{\widetilde{R}_{3}} & \widetilde{R}_{1} & \frac{1}{\widetilde{R}_{1}} & \frac{1}{\widetilde{R}_{1}} & \frac{1}{\widetilde{R}_{3}} & 1,1,1 & \frac{1}{\widetilde{R}_{1}} \\
1,1,1 & \frac{1}{\widetilde{R}_{2}} & 1,1,1 & 1,1,1 & \frac{1}{\widetilde{R}_{1}} & \widetilde{R}_{2} & \widetilde{R}_{1} & \frac{1}{\widetilde{R}_{1}} & \frac{1}{\widetilde{R}_{1}} & \frac{1}{\widetilde{R}_{1}} & \frac{1}{\widetilde{R}_{1}} \\
\widetilde{R}_{1} & \widetilde{R}_{1} & 1,1,1 & 1,1,1 & \frac{1}{\widetilde{R}_{2}} & \widetilde{R}_{2} & \frac{1}{\widetilde{R}_{1}} & \widetilde{R}_{1} & \frac{1}{\widetilde{R}_{1}} & \widetilde{R}_{2} & \frac{1}{\widetilde{R}_{1}} \\
\widetilde{R}_{2} & \widetilde{R}_{3} & \widetilde{R}_{1} & \widetilde{R}_{2} & 1,1,1 & \widetilde{R}_{2} & \frac{1}{\widetilde{R}_{1}} & \widetilde{R}_{1} & \frac{1}{\widetilde{R}_{1}} & \widetilde{R}_{3} & \widetilde{R}_{1} \\
1,1,1 & \frac{1}{\widetilde{R}_{1}} & \frac{1}{\widetilde{R}_{2}} & \frac{1}{\widetilde{R}_{2}} & \frac{1}{\widetilde{R}_{2}} & 1,1,1 & \frac{1}{\widetilde{R}_{2}} & \frac{1}{\widetilde{R}_{2}} & \frac{1}{\widetilde{R}_{1}} & \frac{1}{\widetilde{R}_{1}} & \frac{1}{\widetilde{R}_{2}} \\
\widetilde{R}_{2} & \widetilde{R}_{1} & \frac{1}{\widetilde{R}_{1}} & \widetilde{R}_{1} & \widetilde{R}_{1} & \widetilde{R}_{2} & 1,1,1 & \frac{1}{\widetilde{R}_{1}} & \frac{1}{\widetilde{R}_{1}} & \widetilde{R}_{1} & \frac{1}{\widetilde{R}_{1}} \\
\widetilde{R}_{1} & \widetilde{R}_{1} & \widetilde{R}_{1} & \frac{1}{\widetilde{R}_{1}} & \frac{1}{\widetilde{R}_{1}} & \widetilde{R}_{2} & \widetilde{R}_{1} & 1,1,1 & \frac{1}{\widetilde{R}_{1}} & \widetilde{R}_{1} & \frac{1}{\widetilde{R}_{1}} \\
\widetilde{R}_{3} & \widetilde{R}_{3} & \widetilde{R}_{1} & \widetilde{R}_{1} & \widetilde{R}_{1} & \widetilde{R}_{2} & \widetilde{R}_{1} & \widetilde{R}_{1} & 1,1,1 & \widetilde{R}_{1} & \widetilde{R}_{1} \\
\widetilde{R}_{1} & 1,1,1 & \widetilde{R}_{1} & \frac{1}{\widetilde{R}_{2}} & \frac{1}{\widetilde{R}_{3}} & \widetilde{R}_{1} & \frac{1}{\widetilde{R}_{1}} & \frac{1}{\widetilde{R}_{1}} & \frac{1}{\widetilde{R}_{1}} & 1,1,1 & \frac{1}{\widetilde{R}_{4}} \\
\widetilde{R}_{1} & \widetilde{R}_{1} & \widetilde{R}_{1} & \widetilde{R}_{1} & \frac{1}{\widetilde{R}_{1}} & \widetilde{R}_{2} & \widetilde{R}_{1} & \widetilde{R}_{1} & \frac{1}{\widetilde{R}_{1}} & \widetilde{R}_{4} & 1,1,1
\end{array}\right] .
$$

By applying the procedure of the proposed fuzzy TOPSIS algorithm (Step 1 to Step 2) the vector of weights of business processes and vector of weights of organizational resilience factors with respect to each business process are calculated as follows:

$$
\begin{aligned}
& W_{p}=(0.291,0.139,0.092,0.198,0.250,0.029) \text {, }
\end{aligned}
$$

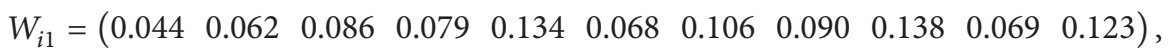

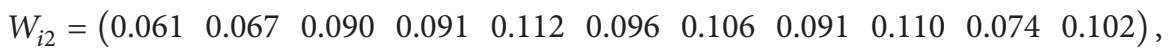

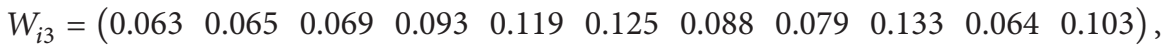

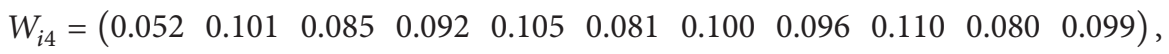

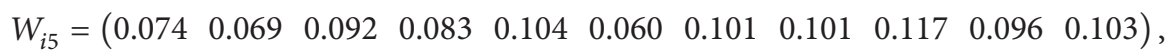

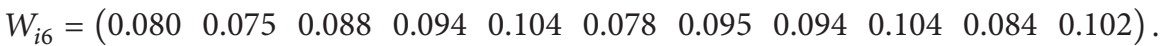

The fuzzy rating values of resilience factors on the level of each business process are presented in Table 1. The weighted decision matrix $\widetilde{F}$ is constructed by applying the proposed Algorithm (Step 3 to Step 5). The values of fuzzy decision matrix $\widetilde{F}$ are presented in Table 2 .

The closeness coefficient for each organizational resilience factor and the rank of the organizational resilience factors are determined by using the proposed algorithm (Step 6 to Step 7). The given results are presented in Table 3.
Organizational resilience potential factors have to be monitored and improved over time because, in a time of disturbance occurring, their decreased level may lead to business catastrophe. An assessment of the organizational resilience in any organization represents an input for determining the strategy of long-term sustainability because in a time of crisis an organization must show that it is capable of functioning despite the evident negative impact. Priority of taking measures which should lead to the improvement 
TABLE 1: Assessed values of the organizational resilience factors.

\begin{tabular}{|c|c|c|c|c|c|c|}
\hline & $p=1$ & $p=2$ & $p=3$ & $p=4$ & $p=5$ & $p=6$ \\
\hline$i=1$ & $\widetilde{v}_{7}$ & $\widetilde{v}_{5}$ & $\widetilde{v}_{6}$ & $\widetilde{v}_{5}$ & $\widetilde{v}_{3}$ & $\widetilde{v}_{2}$ \\
\hline$i=2$ & $\widetilde{v}_{4}$ & $\widetilde{v}_{4}$ & $\widetilde{v}_{4}$ & $\widetilde{v}_{3}$ & $\widetilde{v}_{7}$ & $\widetilde{v}_{2}$ \\
\hline$i=3$ & $\tilde{v}_{3}$ & $\widetilde{v}_{4}$ & $\widetilde{v}_{6}$ & $\widetilde{v}_{3}$ & $\widetilde{v}_{5}$ & $\widetilde{v}_{5}$ \\
\hline$i=4$ & $\tilde{v}_{6}$ & $\widetilde{v}_{5}$ & $\widetilde{v}_{5}$ & $\widetilde{v}_{3}$ & $\widetilde{v}_{4}$ & $\widetilde{v}_{3}$ \\
\hline$i=5$ & $\widetilde{v}_{5}$ & $\widetilde{v}_{6}$ & $\widetilde{v}_{4}$ & $\widetilde{v}_{5}$ & $\widetilde{v}_{5}$ & $\widetilde{v}_{3}$ \\
\hline$i=6$ & $\widetilde{v}_{4}$ & $\widetilde{v}_{4}$ & $\widetilde{v}_{7}$ & $\widetilde{v}_{4}$ & $\widetilde{v}_{5}$ & $\widetilde{v}_{3}$ \\
\hline$i=7$ & $\widetilde{v}_{4}$ & $\widetilde{v}_{4}$ & $\widetilde{v}_{5}$ & $\widetilde{v}_{4}$ & $\widetilde{v}_{5}$ & $\widetilde{v}_{1}$ \\
\hline$i=8$ & $\widetilde{v}_{4}$ & $\widetilde{v}_{3}$ & $\widetilde{v}_{5}$ & $\widetilde{v}_{4}$ & $\widetilde{v}_{5}$ & $\widetilde{v}_{3}$ \\
\hline$i=9$ & $\widetilde{v}_{6}$ & $\widetilde{v}_{4}$ & $\widetilde{v}_{4}$ & $\widetilde{v}_{6}$ & $\widetilde{v}_{5}$ & $\widetilde{v}_{3}$ \\
\hline$i=10$ & $\widetilde{v}_{4}$ & $\widetilde{v}_{4}$ & $\widetilde{v}_{3}$ & $\widetilde{v}_{4}$ & $\widetilde{v}_{5}$ & $\widetilde{v}_{5}$ \\
\hline$i=11$ & $\widetilde{v}_{6}$ & $\widetilde{v}_{4}$ & $\widetilde{v}_{4}$ & $\widetilde{v}_{3}$ & $\widetilde{v}_{6}$ & $\widetilde{v}_{5}$ \\
\hline
\end{tabular}

TABLE 2: The weighted fuzzy decision matrix.

\begin{tabular}{|c|c|c|c|}
\hline & $p=1,10^{-3}$ & $p=2,10^{-3}$ & $p=3,10^{-3}$ \\
\hline$i=1$ & $(0.035,0.040,0.044,0.044)$ & $(0.034,0.043,0.049,0.058)$ & $(0.044,0.050,0.057,0.063)$ \\
\hline$i=2$ & $(0.016,0.025,0.031,0.040)$ & $(0.017,0.027,0.033,0.044)$ & $(0.016,0.026,0.032,0.042)$ \\
\hline$i=3$ & $(0.013,0.026,0.034,0.047)$ & $(0.022,0.036,0.045,0.058)$ & $(0.048,0.055,0.062,0.069)$ \\
\hline$i=4$ & $(0.055,0.063,0.071,0.079)$ & $(0.050,0.064,0.073,0.086)$ & $(0.051,0.065,0.074,0.088)$ \\
\hline$i=5$ & $(0.074,0.094,0.107,0.127)$ & $(0.078,0.090,0.101,0.112)$ & $(0.030,0.048,0.060,0.077)$ \\
\hline$i=6$ & $(0.017,0.027,0.034,0.044)$ & $(0.024,0.038,0.048,0.062)$ & $(0.100,0.112,0.125,0.125)$ \\
\hline$i=7$ & $(0.026,0.042,0.053,0.069)$ & $(0.026,0.042,0.053,0.069)$ & $(0.048,0.062,0.070,0.084)$ \\
\hline$i=8$ & $(0.022,0.036,0.045,0.058)$ & $(0.014,0.027,0.036,0.050)$ & $(0.043,0.055,0.063,0.075)$ \\
\hline$i=9$ & $(0.097,0.110,0.124,0.138)$ & $(0.027,0.044,0.055,0.072)$ & $(0.033,0.053,0.066,0.086)$ \\
\hline$i=10$ & $(0.017,0.028,0.034,0.045)$ & $(0.018,0.030,0.037,0.048)$ & $(0.010,0.019,0.026,0.035)$ \\
\hline \multirow[t]{2}{*}{$i=11$} & $(0.086,0.098,0.111,0.123)$ & $(0.026,0.041,0.051,0.066)$ & $(0.026,0.041,0.051,0.067)$ \\
\hline & $p=4,10^{-3}$ & $p=5,10^{-3}$ & $p=6,10^{-3}$ \\
\hline$i=1$ & $(0.029,0.036,0.042,0.049)$ & $(0.011,0.022,0.030,0.041)$ & $(0,0.008,0.016,0.024)$ \\
\hline$i=2$ & $(0.015,0.030,0.040,0.056)$ & $(0.055,0.062,0.069,0.069)$ & $(0,0.007,0.015,0.022)$ \\
\hline$i=3$ & $(0.013,0.025,0.034,0.047)$ & $(0.051,0.064,0.074,0.087)$ & $(0.048,0.062,0.070,0.084)$ \\
\hline$i=4$ & $(0.014,0.028,0.037,0.051)$ & $(0.021,0.033,0.041,0.054)$ & $(0.014,0.028,0.038,0.052)$ \\
\hline$i=5$ & $(0.058,0.073,0.084,0.100)$ & $(0.057,0.073,0.083,0.099)$ & $(0.016,0.031,0.042,0.057)$ \\
\hline$i=6$ & $(0.020,0.032,0.040,0.053)$ & $(0.033,0.042,0.048,0.057)$ & $(0.012,0.023,0.031,0.043)$ \\
\hline$i=7$ & $(0.025,0.040,0.050,0.065)$ & $(0.056,0.071,0.081,0.096)$ & $(0,0,0.009,0.019)$ \\
\hline$i=8$ & $(0.024,0.038,0.048,0.062)$ & $(0.056,0.071,0.081,0.096)$ & $(0.014,0.028,0.038,0.052)$ \\
\hline$i=9$ & $(0.077,0.088,0.099,0.110)$ & $(0.064,0.082,0.094,0.111)$ & $(0.016,0.031,0.042,0.057)$ \\
\hline$i=10$ & $(0.020,0.032,0.040,0.052)$ & $(0.053,0.067,0.077,0.091)$ & $(0.046,0.059,0.067,0.080)$ \\
\hline$i=11$ & $(0.015,0.030,0.040,0.054)$ & $(0.072,0.082,0.093,0.103)$ & $(0.056,0.071,0.082,0.097)$ \\
\hline
\end{tabular}

of long-term sustainability of an organization is based on the rank of organizational resilience factors. In this way, the organizational resources will be saved and used effectively.

The acquired result indicates that the resilience factors with the greatest impact and with the lowest impact on overall organizational resilience of considered SME are planning strategies $(i=1)$ and detection potential $(i=9)$, respectively. It is clear that planning business strategies are a very important activity that should be carried out on the organization level as well as on the business process level. The measures for the identified factor improvement should correspond to the main goals of business strategy planning and deployment. Some of them may be concentrated on setting objectives related to reducing costs and make business activities more profitable. In the treated organization, the procedures for failure detection are well defined and have been applied in everyday operations, so this organizational resilience factor does not need significant improvement.

\section{Conclusion}

The evaluation and ranking of organizational resilience factors are part of the organizational activities which must be treated permanently because in a time of crisis their 
TABLE 3: Rank of organizational resilience factors.

\begin{tabular}{lcc}
\hline $\begin{array}{l}\text { Organizational } \\
\text { resilience factor }\end{array}$ & $\begin{array}{c}\text { The closeness } \\
\text { coefficient }\end{array}$ & Rank \\
\hline$i=1$ & 0.2788 & 11 \\
$i=2$ & 0.2826 & 10 \\
$i=3$ & 0.3144 & 7 \\
$i=4$ & 0.3473 & 5 \\
$i=5$ & 0.4589 & 2 \\
$i=6$ & 0.3141 & 8 \\
$i=7$ & 0.3595 & 4 \\
$i=8$ & 0.3396 & 6 \\
$i=9$ & 0.4713 & 1 \\
$i=10$ & 0.3047 & 9 \\
$i=11$ & 0.4228 & 3 \\
\hline
\end{tabular}

decreased level may lead to catastrophe. The solution to the considered problem has a key impact on achieving and managing organizational sustainability over time.

In this paper, the novel fuzzy TOPSIS for the evaluating and ranking of organizational resilience factors is proposed. In our procedure, the rating of the relative importance of business processes, organizational resilience values, and their relative importance with respect to each business process are performed by decision makers who have used predefined linguistic expressions. These linguistic expressions have been modeled suitably using triangular and trapezoidal fuzzy numbers. The relative importance of business processes and organizational resilience factors with respect to each business process are stated by a fuzzy pairwise comparison matrix.

By using fuzzy AHP, the weights of business processes and the weights of organizational resilience factors with respect to each business process are calculated and represent the input data for the proposed fuzzy TOPSIS. It can be said that the usage of fuzzy AHP weights in fuzzy TOPSIS makes the application more realistic and reliable. The fuzzy decision matrix is converted into a fuzzy weighted decision matrix and a weighted fuzzy decision matrix is constructed with respect to the organizational resilience factor's type. FPIS and FNIS are determined by using the most commonly used technique for benefit oriented TrFNs, so that FPIS $=(1,1,1,1)$ and FNIS $=(0,0,0,0)$. The fuzzy distance value was used for defuzzification, determining FPIS and FNIS as the crisp values. The closeness coefficient values are calculated by using the procedure which is proposed in the conventional TOPSIS. The higher value of the closeness coefficient indicates that an organizational resilience factor is closer to FPIS and further from FNIS. These values are sorted in decreasing order. The rank of organizational resilience factors corresponds to the rank of the closeness coefficient values. The proposed procedure is illustrated by real-life data from the process industry. The outputs of the proposed novel fuzzy TOPSIS represent promising results for this real case study and this validates its efficiency.

\section{Conflict of Interests}

The authors declare that there is no conflict of interests regarding the publication of this paper.

\section{References}

[1] R. Starr, J. Newfrock, and M. Delurey, "Enterprise resilience: managing risk in the networked economy," International Journal of Production Research, vol. 30, pp. 1-10, 2003.

[2] S. Arsovski, M. Pavlović, Z. Arsovski, and Z. Mirović, "An investigation of relation between sustainable development and quality of life," International Journal for Quality Research, vol. 3, no. 4, pp. 327-337, 2009.

[3] S. Somers, "Measuring resilience potential: an adaptive strategy for organizational crisis planning," Journal of Contingencies and Crisis Management, vol. 17, no. 1, pp. 12-23, 2009.

[4] P. Wymenga, V. Spanikova, A. Barker, J. Konings, and A. Canton, EU SMEs in 2012: At the Crossroads Annual Report on Small and Medium-Sized Enterprises in the EU, Ecorys, Rotterdam, The Netherlands, 2012.

[5] Y. Lee, J. Shin, and Y. Park, “The changing pattern of SME's innovativeness through business model globalization," Technological Forecasting and Social Change, vol. 79, no. 5, pp. 832-842, 2012.

[6] R. Bhamra, S. Dani, and K. Burnard, "Resilience: the concept, a literature review and future directions," International Journal of Production Research, vol. 49, no. 18, pp. 5375-5393, 2011.

[7] G. J. Klir and T. A. Folger, Fuzzy Sets, Uncertainty and Informations, Prentice-Hall, Englewood Cliffs, NJ, USA, 1988.

[8] H. J. Zimmermann, Fuzzy Set Theory and Its Applications, Kluwer Nijhoff Publishing, Boston, Mass, USA, 2001.

[9] T. L. Saaty, "How to make a decision: the analytic hierarchy process," European Journal of Operational Research, vol. 48, no. 1, pp. 9-26, 1990.

[10] C. L. Hwang and K. Yoon, Multiple Attribute Decision Making: Methods and Applications, Springer, Berlin, Germany, 1981.

[11] F. Torfi, R. Z. Farahani, and S. Rezapour, "Fuzzy AHP to determine the relative weights of evaluation criteria and Fuzzy TOPSIS to rank the alternatives," Applied Soft Computing Journal, vol. 10, no. 2, pp. 520-528, 2010.

[12] D. Tadić, D. D. Milanovic, M. Misita, and B. Tadic, "New integrated approach to the problem of ranking and supplier selection under uncertainties," Proceedings of the Institution of Mechanical Engineers B, vol. 225, no. 9, pp. 1713-1724, 2011.

[13] G. Zheng, N. Zhu, Z. Tian, Y. Chen, and B. Sun, "Application of a trapezoidal fuzzy AHP method for work safety evaluation and early warning rating of hot and humid environments," Safety Science, vol. 50, no. 2, pp. 228-239, 2012.

[14] P. V. R. Carvalho, I. L. dos Santos, J. O. Gomes, and M. R. S. Borges, "Micro incident analysis framework to assess safety and resilience in the operation of safe critical systems: a case study in a nuclear power plant," Journal of Loss Prevention in the Process Industries, vol. 21, no. 3, pp. 277-286, 2008.

[15] A. Kelemenis and D. Askounis, "A new TOPSIS-based multicriteria approach to personnel selection," Expert Systems with Applications, vol. 37, no. 7, pp. 4999-5008, 2010.

[16] H.-S. Shih, H.-J. Shyur, and E. S. Lee, "An extension of TOPSIS for group decision making," Mathematical and Computer Modelling, vol. 45, no. 7-8, pp. 801-813, 2007.

[17] T. Yang and C.-C. Hung, "Multiple-attribute decision making methods for plant layout design problem," Robotics and 
Computer-Integrated Manufacturing, vol. 23, no. 1, pp. 126-137, 2007.

[18] I. Mahdavi, N. Mahdavi-Amiri, A. Heidarzade, and R. Nourifar, "Designing a model of fuzzy TOPSIS in multiple criteria decision making," Applied Mathematics and Computation, vol. 206, no. 2, pp. 607-617, 2008.

[19] S. Sadi-Nezhad and K. K. Damghani, "Application of a fuzzy TOPSIS method base on modified preference ratio and fuzzy distance measurement in assessment of traffic police centers performance," Applied Soft Computing Journal, vol. 10, no. 4, pp. 1028-1039, 2010.

[20] C. Chakraborty and D. Chakraborty, "A theoretical development on a fuzzy distance measure for fuzzy numbers," Mathematical and Computer Modelling, vol. 43, no. 3-4, pp. 254261, 2006.

[21] S. S. Nezhad and R. G. Assadi, "Preference ratio-based maximum operator approximation and its application in fuzzy flow shop scheduling," Applied Soft Computing Journal, vol. 8, no. 1, pp. 759-766, 2008.

[22] A. T. Gumus, "Evaluation of hazardous waste transportation firms by using a two step fuzzy-AHP and TOPSIS methodology," Expert Systems with Applications, vol. 36, no. 2, pp. 40674074, 2009.

[23] D.-Y. Chang, "Applications of the extent analysis method on fuzzy AHP," European Journal of Operational Research, vol. 95, no. 3, pp. 649-655, 1996.

[24] S. M. Baas and H. Kwakernaak, "Rating and ranking of multipleaspect alternatives using fuzzy sets," Automatica, vol. 13, no. 1, pp. 47-58, 1977.

[25] G. Işiklar and G. Büyüközkan, "Using a multi-criteria decision making approach to evaluate mobile phone alternatives," Computer Standards and Interfaces, vol. 29, no. 2, pp. 265-274, 2007.

[26] B. Sadeghpour-Gildeh and D. Gien, "La Distance-Dpq et le coeffcient de orrelation entre deux variables aleatories floues," Actes de LFA, vol. 2001, pp. 97-102, 2001.

[27] J. Oakland, Oakland on Quality Management, Elsevier, Butterworth-Heinemann, 2004.

[28] A. Lee, J. Vargo, and E. Seville, "Developing a tool to measure and compare organizations resilience," Natural Hazards Review, vol. 14, pp. 29-41, 2013.

[29] A. Aleksić, M. Stefanović, S. Arsovski, and D. Tadić, "An assessment of organizational resilience potential in SMEs of the process industry, a fuzzy approach," Journal of Loss Prevention in the Process Industries, 2013.

[30] H. J. Zimmermann, "Intelligent decision support system," in Proceedings of the European Symposiun on Intelligent Techniques, 23924, Aachen, Germany, 1997.

[31] H. J. Zimmermann, "Results of empirical studies in fuzzy set theory," in Applied General Systems Research, G. J. Klir, Ed., pp. 303-311, Plenum Publishing Corporation, 1978.

[32] R. C. Berkan and S. L. Trubatch, Fuzzy Variables Design in Fuzzy Systems Design Principles: Building Fuzzy IF-THEN Rules Bases, Wiley-IEEE Press, 1997.

[33] D. Petrović, Modelling and Analysis of Serial Supply Chains in Uncertain Environments [Ph.D. dissertation], Department of Engineering, University of Warwick, 1997.

[34] G. Klir and B. Yuan, Fuzzy Sets and Fuzzy Logic, Theory and Applications, Prentice Hall, 1995.

[35] F. A. Lootsma, Fuzzy Logic for Planning and Decision-Making, Kluwer Academic Publishers, Boston, Mass, USA, 1997.
[36] D. Dubois and H. Prade, Fuzzy Sets and Systems: Theory and Applications, Academic press, London, UK, 1980.

[37] G. Bortolan and R. Degani, "A review of some methods for ranking fuzzy subsets," Fuzzy Sets and Systems, vol. 15, no. 1, pp. 1-19, 1985.

[38] C. K. Kwong and H. Bai, "Determining the importance weights for the customer requirements in QFD using a fuzzy AHF with an extent analysis approach," IIE Transactions, vol. 35, no. 7, pp. 619-626, 2003.

[39] T.-C. Wang and T.-H. Chang, "Application of TOPSIS in evaluating initial training aircraft under a fuzzy environment," Expert Systems with Applications, vol. 33, no. 4, pp. 870-880, 2007. 


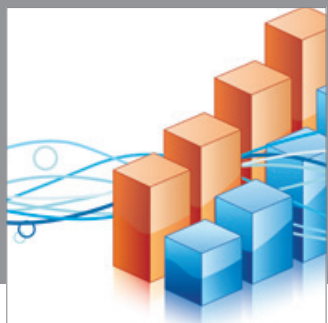

Advances in

Operations Research

mansans

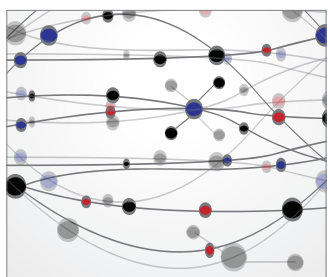

The Scientific World Journal
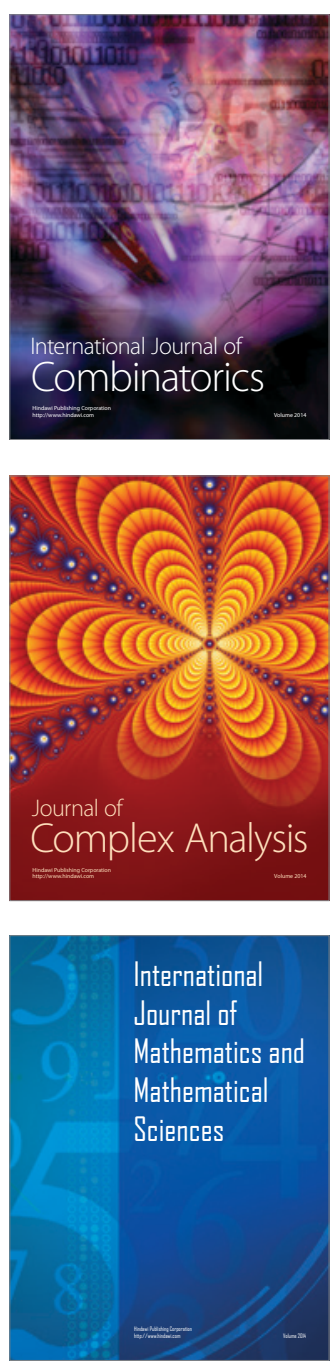
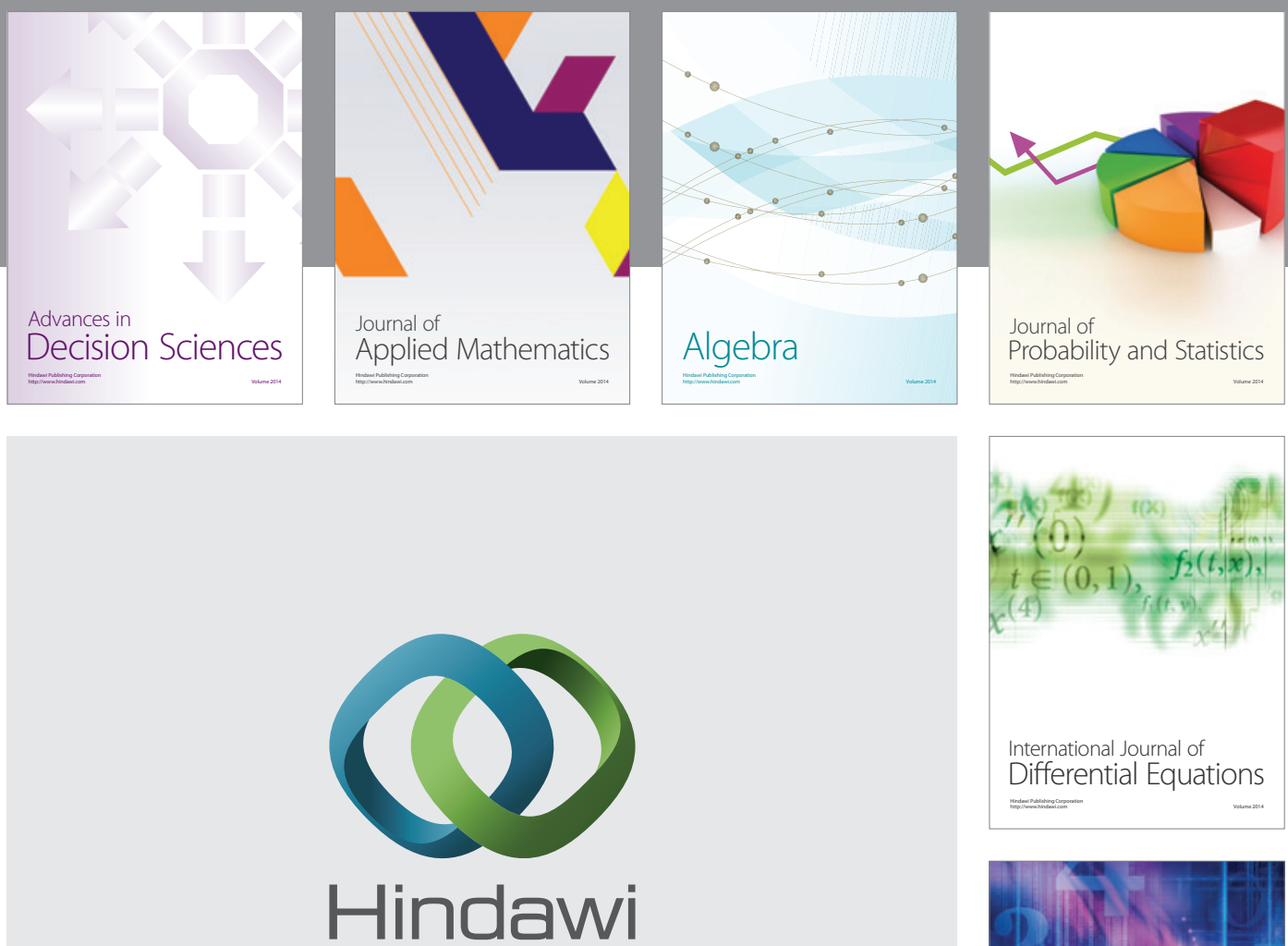

Submit your manuscripts at http://www.hindawi.com
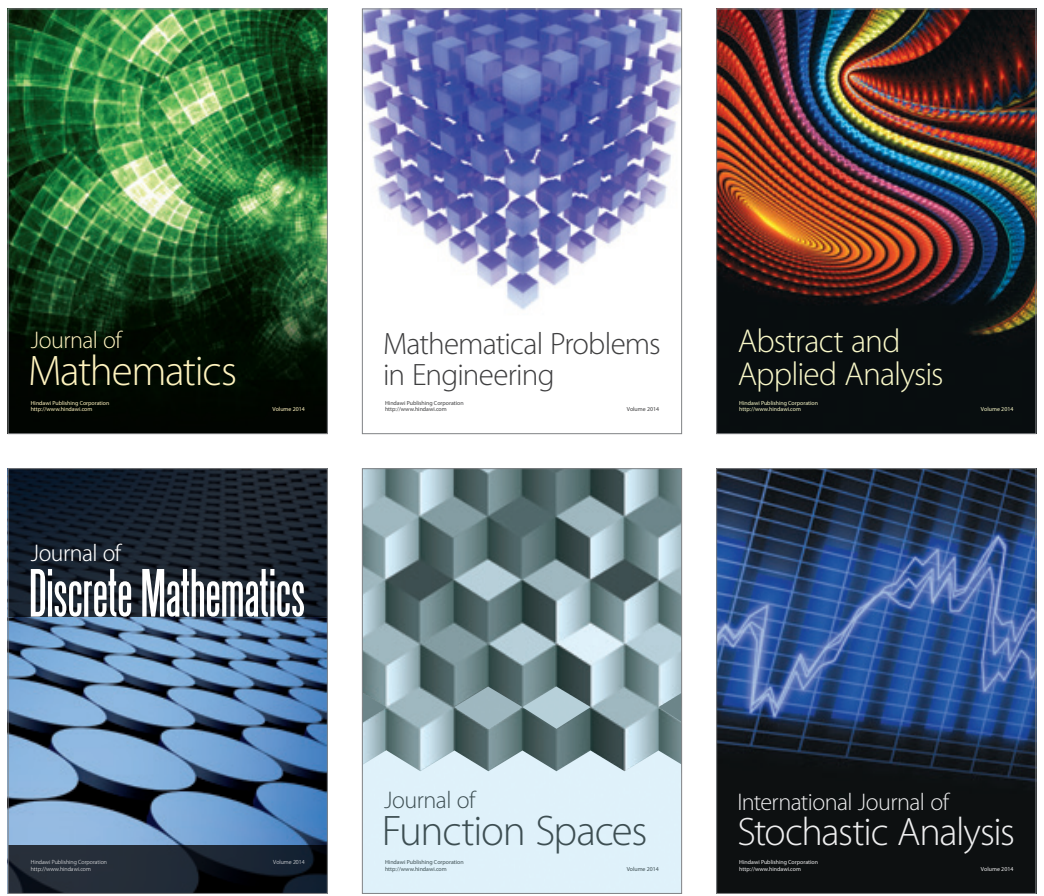

Journal of

Function Spaces

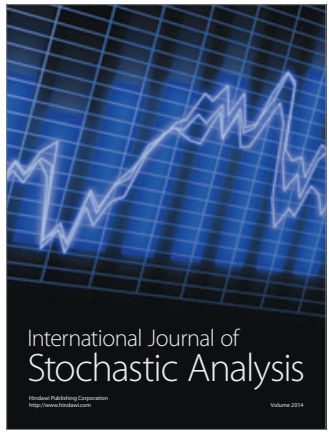

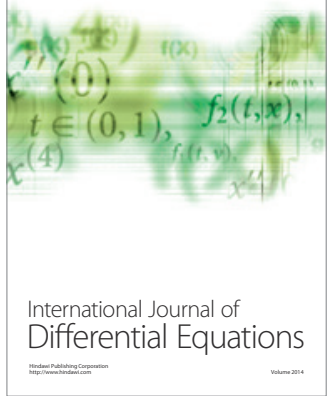
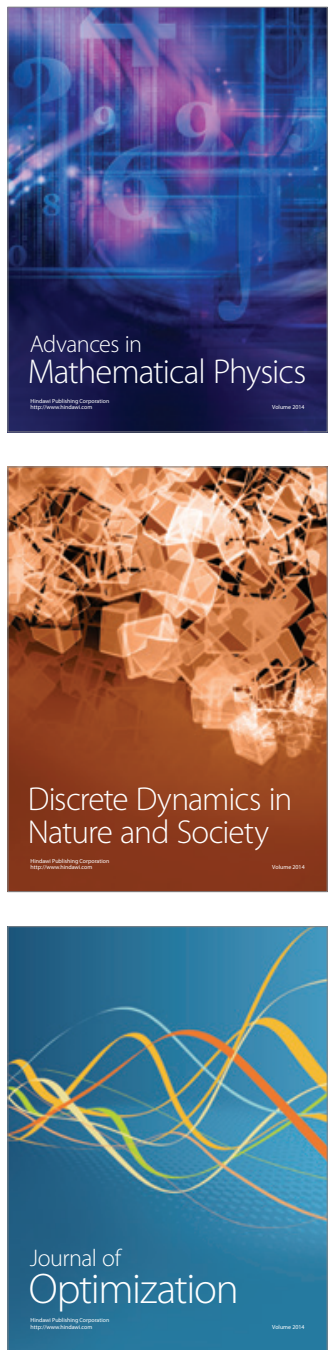